\title{
ANCA associated vasculitis: The Journey to complement-targeted therapies.
}

Luis F. Quintana 1, Andreas Kronbichler 2, Miquel Blasco 1, Ming-hui Zhao ${ }^{3}$ David Jayne ${ }^{4}$

1 Department of Nephrology and Renal Transplantation, Hospital Clínic, Centro de Referencia en Enfermedad Glomerular Compleja del Sistema Nacional de Salud (CSUR), Department of Medicine, University of Barcelona, IDIBAPS, Barcelona, Spain

2 Department of Internal Medicine IV (Nephrology and Hypertension), Medical University Innsbruck, Innsbruck, Austria

${ }^{3}$ Renal Division, Peking University First Hospital, No. 8 Xishiku Street, Beijing 100034, China.

4Vasculitis and Lupus Clinic, Addenbrooke's Hospital. Department of Medicine, University of Cambridge, Hills Road, CB2 0QQ, Cambridge, United Kingdom

word cout 2940

*Correspondence should be addressed to:

Luis F. Quintana, MD, PhD, Department of Nephrology and Renal Transplantation, Hospital Clínic, Department of Medicine, University of Barcelona, Barcelona, Spain.

email: Ifquinta@clinic.cat 
Abstract

ANCA associated vasculitis is a serious, very often recurrent disease that despite the current standard treatment with high-dose glucocorticoids and either cyclophosphamide or rituximab, patients have a nine-fold increased mortality risk in the first year compared with healthy controls, attributed to infections, vasculitis activity, and renal disease.

During the last few years, novel findings have suggested that activation of the complement system, in particular the alternative complement system, has a significant role in ANCA associated vasculitis pathogenesis. Detection of several components of this system in the circulation and urine reflects disease activity, and thus may be useful for clinical prognosis and to set up personalised treatments. In fact, some components of the complement system, such as $\mathrm{C} 5 \mathrm{a}$, might be potential targets for therapy.

In this Review an update on clinical evidence for the role of complement activation in AAV is provided and subsequently we discuss potential therapeutic strategies that target complement components and open the way for clinical use of this target therapy in the near future. 


\section{Introduction}

The spectrum of anti-neutrophil cytoplasm antibody (ANCA)-associated vasculitis (AAV) includes three clinical phenotypes: granulomatosis with polyangiitis (GPA, Wegener's granulomatosis), microscopic polyangiitis (MPA) and eosinophilic granulomatosis with polyangiitis (EGPA, Churg-Strauss syndrome). The availability of ANCA as a biomarker eases diagnostics and provides prognostic information. A cytoplasmic pattern (c-ANCA) and the target autoantigen proteinase 3 (PR3) are predominantly present in GPA (40-50\% in localised GPA, $70-95 \%$ in generalised cases), while the perinuclear pattern (p-ANCA) and presence of myeloperoxidase (MPO) are observed in most MPA cases (70-95\%) and to a lesser extent in EGPA (around 40\%) $(1,2)$. GWAS identified MHC and non-MHC associations with AAV and genetic associations better correlated with the ANCA serotype than with the clinical phenotype, supporting the concept that PR3 ANCA-and MPO ANCAassociated vasculitis are distinct autoimmune syndromes and should replace classification based on the clinical phenotype (3).

$A A V$ is a serious, very often recurrent disease that despite the current standard treatment with high-dose glucocorticoids and either cyclophosphamide or rituximab, patients have a nine-fold increased mortality risk in the first year compared with healthy controls, attributed to infections, vasculitis activity, and renal disease (4). The prevalence of kidney involvement in AAV is $75-90 \%$, and also impacts patients' quality of life and morbidity, particularly among those with end stage renal disease (ESRD) (4). Moreover, a histological subgrouping of diagnostic renal biopsies has been proposed to aid in prognosis (5). Renal biopsy and baseline estimated glomerular filtration rate (eGFR) remain the most important outcome predictors available for ANCA associated renal vasculitis. The severity of proteinuria after the diagnosis is also a marker of the degree of injury and MPO-ANCA positive patients have a worse renal prognosis due to more severe chronic damage at the time of diagnosis (6). 
Currently used therapies contribute to more than half of the increased risk of mortality and represent an important cause of comorbidities. Among them, glucocorticoids are associated with an increased infection risk and increase the risk to develop cardiovascular complications, particularly hypertension and diabetes mellitus (7-12).

The histopathological hallmark in the renal biopsy of patients with AAV is pauci-immune necrotising crescentic glomerulonephritis, which is characterised by no immunoglobulin and complement deposition in the glomeruli. In addition, hypocomplementemia is infrequent in patients with AAV.(4) These characteristics diverted the attention on a potential role of the complement system in the development of AAV over decades. However, gathering evidence during the last few years has suggested that activation of the complement system, in particular the alternative complement system, has a significant role in AAV pathogenesis. Detection of several components of this system in the circulation and urine reflect disease activity, and thus may be useful for clinical prognosis and to set up personalised treatments. In fact, some components of the complement system, such as C5a, might be potential targets for therapy.

In this Review an update on clinical evidence for the role of complement activation in AAV is provided and subsequently we discuss potential therapeutic strategies that target complement components and could improve patient and renal outcomes in this clinical context.

\section{Complement and Glomerular disease}

The complement system and its regulatory proteins are components of the innate immune system with multiple roles in glomerulonephritis (GN) (13-17). The innate immune response involves immediate complement activation through the mannose binding lectin (MBL) or alternative pathways (13-16). Activation of the MBL pathway proceeds when MBL 
binds to mannose residues on pathogens and activates the serine proteases, MASP-1 and MASP-2, leading to activation of C4 and C2. The alternative pathway is activated spontaneously by hydrolysis of C3 and amplified by defects in complement regulation. Non-Ig zymogens such as damaged cells and bacterial and viral proteins can also activate the alternative pathway beginning directly at C3. The same initiating event may activate more than one pathway (17).

Complement activation products are the main mediators of antibody-induced GN. Usually this involves $\mathrm{C} 1 \mathrm{q}$ binding to Immunoglobulins that leads to classic pathway activation through $\mathrm{C} 4$ and $\mathrm{C} 2$; however, some immunoglobulins, depending on their level of glycosylation, can also bind MBL. IgG subclasses 1 and 3 and IgM are classic complement pathway activators, whereas $\lg G 2$ and $4, \lg A$, $\lg D$, and $\lg E$ activate complement poorly (16-17).

All complement activation pathways proceed through cleavage of C3 and C5 leading to release of chemotactic factors such as $\mathrm{C} 5 \mathrm{a}$ that attract inflammatory cells (neutrophils, macrophages, and platelets) when abutting the circulation as well as formation of the terminal membrane attack complex (C5b-9) (13-15).

Sublytic quantities of C5b-9 can insert into lipid bilayers of adjacent glomerular cell membranes, initiate several signaling pathways, and convert these cells to effector cells which may proliferate; release a variety of cytokines, growth factors, eicosanoids, oxidants, proteases, and other acute inflammatory mediators; as well as up-regulate production of matrix components that contribute to chronic scarring and sclerosis.(18-20) Complement activation products like C5a can also activate toll-like receptors (TLRs).(20) Complement activation in vivo is tightly regulated by a number of circulating and cell-bound complement regulatory proteins, whose functions, mutations and deficiencies are also important in the development of several glomerular diseases. Abnormalities in serum complement profiles are in some cases helpful in assessing the nature of the underlying disease and its activity, 
but significant complement-mediated injury may occur locally without alterations in circulating complement components.(13-14)

\section{Complement activation in the AAV pathogenesis}

Until recently, the complement system was not studied in AAV because of its pauciimmune nature with absence of complement deposition in affected tissues and its rare association with hypocomplementemia. (21-22) The first evidence for a role of complement in AAV pathogenesis came from observations in animal studies. These models demonstrated that the alternative complement pathway was essential to the development of a necrotising crescentic GN.(23) Complement-depleted mice pretreated with cobra venom factor were protected from the development of $\mathrm{GN}$, as evidenced after the induction of a pauci-immune crescentic GN by the administration of anti-MPO splenocytes to the mice. In these models, complement depletion prevented inflammation and crescent formation. Moreover, other studies clearly defined the contribution of the complement system to AAV pathogenesis. C5-knockout mice aiming to block activation of the terminal complement pathway were also protected as were mice lacking complement factor $\mathrm{B}$, a critical regulatory component of the alternative complement pathway. By the contrary, C4knockout mice developed the disease. In brief, these data confirm that the alternative pathway and the activation of complement C5 and the terminal complement pathway are critical to AAV pathogenesis.

Furthermore, supernatant from human neutrophils stimulated with either anti-MPO or PR3 IgG activated complement in normal human serum, prompting to the release of a complement-activating product by the ANCA-stimulated neutrophils. (23) 
Additionally, pretreatment with a monoclonal antibody blocking C5 avoided the development of GN in mice and the lesion may be halted when given to mice after GN had been established. (24)

Animal data were interpreted with caution given the important differences between mice model and men, and consequently generated the reassessment of human data. In agreement with the results generated by animal models, this reevaluation confirmed that there were some clues that complement activation plays a role in human AAV. (25) Despite that the intensity of the staining is not as bright as other observed in diverse GNs, renal biopsies of AAV patients do stain positive for some complement activation proteins.(22) Lately, investigation of renal biopsies of patients with ANCA-associated GN found an association between those biopsies showing presence of immune deposits and proteinuria (26), as well as between alternative pathway proteins and crescent formation $(22,27)$. Other signs of complement activation in human AAV include increased levels of urinary alternative pathway activation products and presence of alternative pathway activation fragments in the serum.(28-29) Moreover, a study confirmed the presence of hypocomplementaemia in a small group of patients with worst overall and renal prognosis.

Finally, a proteomic analysis of renal tissues from AAV patients with ANCA-negative GN showed larger amounts of C3 and C9 deposition compared with ANCA-positive patients suggesting that ANCA-negative AAV was as a separate disease phenotype, promoted by a specific alteration in the activation of the alternative complement pathway. (31)

Since some years, the role of neutrophils in complement activation (both classical and alternative pathways) by expelling neutrophil extracellular traps (NETs) was well characterised.(32-33). In AAV, a study reported that neutrophils activated by MPO-ANCA released factors that had the capacity to activate the alternative complement pathway with the involvement of microparticles (34) or NETs (32-35). 
From another point of view, a study showed that complement factor $H$, the main negative regulator of the alternative complement pathway, inhibits neutrophil activation by ANCA. Nonetheless, factor $H$ from patients with active AAV was deficient in its ability to bind neutrophils and inhibit their activation suggesting that the complement system regulators may also play a protective role in neutrophil activation in AAV. (36)

Even more, complement activation in AAV may not only result in neutrophil degranulation, but may also impact on the coagulation cascade. The effect of C5a on both neutrophils and endothelial cells to upregulate tissue factor and initiate the extrinsic pathway of coagulation is well known (37). Platelets express C5a receptors and activated platelets may in turn push on complement and promote a vicious circle of neutrophils degranulation, coagulation, and lingering complement activation. (38)

\section{Alternative complement pathway profile for disease stratification}

In 2015, Manenti et al. reported, in a study that included 46 patients with AAV, that $35 \%$ of these individuals had decreased levels of $\mathrm{C} 3$ in serum, and this finding was associated with decreased renal survival and the presence of renal thrombotic microangiopathy in the biopsy (39). Recently, a study in 76 AVV patients reported that only $5 \%$ had hypocomplementaemia, however, this finding implied worst prognosis with lower overall and renal survival in this subset of individuals (30).

Moreover, the plasma level of properdin in patients with AAV in active stage was significantly lower than that in normal controls. The level of properdin inversely correlated with the proportion of crescents in renal specimens of AAV. (29)

Urine Levels of $\mathrm{Bb}, \mathrm{C} 3 \mathrm{a}, \mathrm{C} 5 \mathrm{a}$ and $\mathrm{C} 5 \mathrm{~b}-9$ were higher in patients with active AAV than in patients in remission and levels of $\mathrm{Bb}$ correlated directly with serum creatinine, and inversely with the proportion of normal glomeruli in renal biopsies.(40) 
Complement deposits in renal tissue may also be a marker of disease severity. Their presence has been correlated with worst renal function and proteinuria at presentation; and a higher proportion of glomerular crescents and severe tubulointerstitial injury $(22,27,41)$. In fact, C3c deposition was found in renal biopsies and was associated with poorer renal function. C5b-9, C3d, and factor B, but not C4d, could be detected in active glomerular lesions and C3d and properdin staining was associated with the proportion of cellular crescents, confirming the role of C3d-staining as an independent risk factor for the development of ESRD (42)

Expression of the two C5a receptors, C5aR and C5L2, was mainly found on infiltrating neutrophils and macrophages. C5L2 was upregulated, whereas expression of C5aR was downregulated and correlated with serum creatinine levels at diagnosis and the extent of interstitial fibrosis. (39-41)

All these findings in plasma, urine and kidneys of AAV patients provide additional evidence that activation of the alternative complement pathway occurs in the development of AAV and may have a role in clinical stratification of disease severity.

\section{Complement-targeted therapies in AVV}

Current therapeutic options for AAV are based on general immune-suppression administrated as induction treatment with cyclophosphamide or rituximab and steroids. For maintenance treatment, azathioprine, methotrexate and rituximab are recommended. This standard of care has transformed these group of entities from a usually fatal disease into a chronic condition with co-morbidity and high relapse risk. (43)

In this setting, finding new therapies to save steroid doses and prevent recurrence of disease are one of the main priorities. Given the background that complement is actively 
involved in pathogenesis, complement targeting therapies are very attractive option to solve this important clinical issue.(44)

In recent years, drugs have been designed to target specifically one of the activation pathways of the complement cascade; it is possible to avoid blocking complement activation itself, by blocking the detection of activated complement fragments by cellular complement receptors instead. Using specific inhibitors that block one particular aspect of complement activation, the therapeutic result can be reached without affecting the other critical aspects of complement activation and innate immune response. (44)

There are several classes of complement-inhibiting molecules in different phases of development, including therapeutic blocking antibodies, purified human proteins, recombinant proteins from pathogens, peptides or small interfering RNAs. $(25,44)$

Only two types of these drugs are clinically available: the C5-blocking antibody eculizumab (Soliris ${ }^{\circledR}$, Alexion Pharmaceuticals, Boston, Massachusetts, USA) and several C1-inhibitor (C1-INH) preparations (Cinryze ${ }^{\circledR}$, Shire Pharmaceuticals, Dublin, Ireland; Berinert $®$, CSL Behring, King of Prussia, Pennsylvania, USA and Ruconest $($, Pharming Group, Leiden, The Netherlands). The C1-INH preparations are mostly used for the treatment of angiooedema.(45)

Eculizumab has been available for clinical use for more that 10 years in the treatment of paroxysmal nocturnal haematuria $(\mathrm{PNH})$ and atypical haemolytic uremic syndrome (aHUS), with very good outcomes and important information about its use and safety is available elsewhere. (46-48)

Due to differences between PNH and AAV pathogenesis, efforts for complement inhibition in AAV has been focused on inhibiting the signaling of the C5aR. Complement target therapy in AAV has been tested in animal models, blocking C5 activation prior to lesion induction could prevent MPO-ANCA-triggered vasculitis in mice and most of the effect of complement activation was mediated by C5aR1, the receptor for C5a (49-50). 
These observations clearly indicate $\mathrm{C} 5$ and $\mathrm{C} 5 \mathrm{aR}$ as possible therapeutic targets in experimental AAV using a small compound inhibitor of the C5a receptor with dosedependent inhibition effect of MPO-ANCA induced GN. (51)

In line with these observations, there was no major role for the membrane attack complex (C5b-9) in the pathogenesis of AAV. Eculizumab avoids the cleavage of C5 but at the same time completely blocks membrane attack complex (MAC) formation in pathogens, potentially adding an undesirable and unnecessary risk of severe infections in the setting of AAV. (52)

\section{CCX168: The oral C5aR blocker experience in ANCA associated vasculitis}

As a consequence of these bench findings, clinical trials have been launched to test the safety and efficacy of an orally available human $\mathrm{C} 5 \mathrm{aR}$ blocker, the small molecule CCX168 (Avacopan $\AA$, ChemoCentryx, Mountain View, California, USA). The phase I studies revealed a good safety profile, while producing more than $90 \%$ receptor blockade in inflammatory cells in the blood throughout the day.(53)

Next, the C5aR inhibitor on Leukocytes Exploratory ANCA-associated Renal Vasculitis (CLEAR) trial was performed using CCX168. In this randomised, double-blind, placebocontrolled phase II trial, CCX168 was orally administered to AAV patients.(54) Three groups were compared: one receiving the standard treatment (cyclophosphamide/ rituximab and high-dose prednisolone and placebo), one receiving CCX168 and prednisolone (cyclophosphamide/rituximab and low-dose prednisolone and 30mg CCX168 twice daily) and the third group receiving CCX168 without prednisolone (cyclophosphamide/rituximab and no prednisolone and 30mg CCX168 twice daily). At 12 weeks, it was observed that CCX168 was well tolerated with no unexpected adverse events. It was concluded that CCX168 was at least equally effective as the standard of 
care.(54) Following this trial, the Clinical ANCA Vasculitis Safety and Efficacy Study of Inhibitor of C5aR (CLASSIC) was performed. In this dose-ranging Phase II study of CCX168 which was added to standard of care, no safety concerns were reported and a dose-response effect of the treatment was established. (55) Currently, a Phase III Clinical Trial of CCX168 in AAV (ADVOCATE) is ongoing and will assess the proportion of patients achieving remission at 26 and 52 weeks. (56)

\section{Conclusions}

Despite the use of the best available current therapies and achieving high levels of early clinical responses, ESRD caused by ANCA associated GN is an avoidable outcome yet, with rates approaching $40 \%$. Major hurdles to management include the complex balance between toxicity associated with steroids and other immunosuppressive therapies and the risk of recurrence. The recent advances in the understanding of the role of complement in AAV pathogenesis clearly indicate $\mathrm{C} 5$ and $\mathrm{C} 5 \mathrm{aR}$ as possible therapeutic targets in experimental AAV and open the way for clinical use of complement target therapy in the near future. 


\section{References}

1. Millet A, Pederzoli-Ribeil M, Guillevin L, Witko-Sarsat V, Mouthon L. Antineutrophil cytoplasmic antibody-associated vasculitides: is it time to split up the group? Annals of the rheumatic diseases 72(8):1273-9,2013.

2. Greco A, Rizzo MI, De Virgilio A, et al. Churg-Strauss syndrome. Autoimmunity Reviews 14(4): 341-8,2015.

3. Lyons PA, Rayner TF, Trivedi $S$ et al. Genetically distinct subsets within ANCA-associated vasculitis. N Engl J Med 367: 214-223,2012.

4. Franssen CF, Stegeman CA, Kallenberg CG et al. Antiproteinase 3- and antimyeloperoxidase associated vasculitis. Kidney Int 57: 2195-2206,2000.

5. Berden AE, Ferrario F, Hagen EC et al. Histopathologic classification of ANCA-associated glomerulonephritis. J Am Soc Nephrol 21: 1628-1636,2010.

6. Quintana LF, Perez N.S., De sousa E, Rodas LM, et al. ANCA serotype and histopathological classification for the prediction of renal outcome in ANCA-associated glomerulonephritis. Nephrol Dial Transplant 29: 1764-1769,2014.

7. Little MA., Nightingale P, Verburgh CA, Hauser T, De Groot K, Savage C, Jayne D, Harper L; European Vasculitis Study (EUVAS) Group: Early mortality in systemic vasculitis: Relative contribution of adverse events and active vasculitis. Ann Rheum Dis 69: 1036-1043, 2010

8. Jayne D: Evidence-based treatment of systemic vasculitis. Rheumatology (Oxford) 39: 585-595, 2000

9. KoldingsnesW, Nossent $\mathrm{H}$ : Predictors of survival and organ damage in Wegener's granulomatosis. Rheumatology (Oxford) 41: 572-581, 2002

10. Corral-Gudino L, Borao-Cengotita-Bengoa M, Del Pino-Montes J, Lerma-Márquez JL: Overall survival, renal survival and relapse in patients with microscopic polyangiitis: A systematic review of current evidence. Rheumatology (Oxford) 50: 1414-1423, 2011

11. Takala JH, Kautiainen H, Finne P, Leirisalo-Repo M: Wegener's granulomatosis in Finland in 1981-2000: Risk of dialysis-dependent renal disease. Scand J Rheumatol 40: 283-288, 2011

12. Mohammad AJ, Segelmark M: A population-based study showing better renal prognosis for proteinase 3 antineutrophil cytoplasmic antibody (ANCA)-associated nephritis versus myeloperoxidase ANCA associated nephritis. J Rheumatol 41: 1366-1373, 2014

13. Pickering M, Cook HT: Complement and glomerular disease: New insights. Curr Opin Nephrol Hypertens 20: 271-277, 2011

14. Puri TS, Quigg RJ: The many effects of complement C3- and C5-binding proteins in renal injury. Semin Nephrol 27: 321-337, 2007.

15. Lesher AM, Song WC: Review: Complement and its regulatory proteins in kidney diseases. Nephrology (Carlton) 15: 663-675, 2010.

16. Chen M, Daha MR, Kallenberg CG: The complement system in systemic autoimmune disease. J Autoimmun 34: J276-J286, 2010.

17. Daha NA, Banda NK, Roos A, Beurskens FJ, Bakker JM, Daha MR, Trouw LA: Complement activation by (auto-) antibodies. Mol Immunol 48: 1656-1665, 2011.

18. Schroeder HW Jr, Cavacini L: Structure and function of immunoglobulins. J Allergy Clin Immunol 125[Suppl 2]: S41-S52, 2010.

19. Couser WG, Baker PJ, Adler S: Complement and the direct mediation of immune glomerular injury: A new perspective. Kidney Int 28: 879-890, 1985.

20. Stevens MG, Van Poucke M, Peelman LJ, Rainard P, De Spiegeleer B, Rogiers C, Van de Walle GR, Duchateau L, Burvenich C: Anaphylatoxin C5a-induced toll-like receptor 4 signaling in bovine neutrophils. J Dairy Sci 94: 152-164, 2011.

21. Jennette JC, Wilkman AS, Falk RJ. Anti-neutrophil cytoplasmic autoantibody-associated glomerulonephritis and vasculitis. Am J Pathol 135:921-930, 1989.

22. Xing GQ, Chen M, Liu G, Heeringa P, Zhang JJ, Zheng X, E J, Kallenberg CG, Zhao MH. Complement activation is involved in renal damage in human antineutrophil cytoplasmic autoantibody associated pauci-immune vasculitis. J Clin Immunol 29:282-291, 2009.

23. Xiao H, Schreiber A, Heeringa P, Falk RJ, Jennette JC. Alternative complement pathway in the pathogenesis of disease mediated by anti-neutrophil cytoplasmic autoantibodies. Am J Pathol 170:52-64, 2007. 
24. Huugen D, van Esch A, Xiao H, Peutz-Kootstra CJ, Buurman WA, Tervaert JW, Jennette JC, Heeringa P. Inhibition of complement factor C5 protects against anti-myeloperoxidase antibodymediated glomerulonephritis in mice. Kidney Int 71:646-654, 2007.

25. Chen M, Jayne DR, Zhao MH. Complement in ANCA-associated vasculitis: mechanisms and implications for management. Nat Rev Nephrol 13:359-367,2017.

26. Scaglioni V, Scolnik M, Catoggio LJ, et al. ANCA-associated pauci-immune glomerulonephritis: always pauci-immune? Clin Exp Rheumatol; 35 (Suppl 103):55-58,2017.

27. Hilhorst $M$, van Paassen $P$, van Rie $H$, et al. Complement in ANCA-associated glomerulonephritis. Nephrol Dial Transplant; 32:1302-1313, 2017.

28. Gou SJ, Yuan J, Wang C, et al. Alternative complement pathway activation products in urine and kidneys of patients with ANCA-associated GN. Clin J Am Soc Nephrol; 8:1884-1891,2013.

29. Gou SJ, Yuan J, Chen M, et al. Circulating complement activation in patients with antineutrophil cytoplasmic antibody-associated vasculitis. Kidney Int; 83:129-137,2013.

30. Deshayes S, Aouba A, Khoy K, et al. Hypocomplementemia is associated with worse renal survival in ANCA-positive granulomatosis with polyangiitis and microscopic polyangiitis. PLoS One; 13:e0195680, 2018.

31. Sethi S, Zand L, De Vriese AS, et al. Complement activation in pauci-immune necrotizing and crescentic glomerulonephritis: results of a proteomic analysis. Nephrol Dial Transplant; 32(suppl1):i139-i145,2017.

32. Leffler J, Martin M, Gullstrand B, et al. Neutrophil extracellular traps that are not degraded in systemic lupus erythematosus activate complement exacerbating the disease. J Immunol; 188:3522-3531,2012.

33. Wang $\mathrm{H}$, Wang $\mathrm{C}$, Zhao $\mathrm{MH}$, Chen $\mathrm{M}$. Neutrophil extracellular traps can activate alternative complement pathways. Clin Exp Immunol; 181: 518-527, 2015.

34. Camous L, Roumenina L, Bigot S, et al. Complement alternative pathway acts as a positive feedback amplification of neutrophil activation. Blood;117:1340-1349,2011.

35. Schreiber A, Rousselle A, Becker JU, et al. Necroptosis controls NET generation and mediates complement activation, endothelial damage, and autoimmune vasculitis. Proc Natl Acad Sci U S A ; 114:E9618-e9625, 2017.

36. Chen SF, Wang FM, Li ZY, et al. Complement factor $\mathrm{H}$ inhibits anti-neutrophil cytoplasmic autoantibody-induced neutrophil activation by interacting with neutrophils. Front Immunol; 9:559,2018.

37. Kambas K, Chrysanthopoulou A, Vassilopoulos D, et al. Tissue factor expression in neutrophil extracellular traps and neutrophil derived microparticles in antineutrophil cytoplasmic antibody associated vasculitis may promote thromboinflammation and the thrombophilic state associated with the disease. Ann Rheum Dis; 73:1854-1863,2014.

38. Del Conde I, Cruz MA, Zhang H, et al. Platelet activation leads to activation and propagation of the complement system. J Exp Med ; 201:871-879,2005.

39. Manenti, L. et al. Association of serum C3 concentration and histologic signs of thrombotic microangiopathy with outcomes among patients with ANCA-associated renal vasculitis. Clin. J. Am. Soc. Nephrol. 10, 2143-2151,2015.

40. Yuan J, Gou SJ, Huang J, Hao J, Chen M, Zhao MH. C5a and its receptors in human antineutrophil cytoplasmic antibody (ANCA)-associated vasculitis. Arthritis Res Ther 12;14(3):R140,2012.

41. Chen $M$, Xing GQ, Yu F, et al. Complement deposition in renal histopathology of patients with ANCA-associated pauci-immune glomerulonephritis. Nephrol Dial Transplant 24:1247-1252,2009.

42. Villacorta J, Diaz-Crespo F, Acevedo M, et al. Glomerular C3d as a novel prognostic marker for renal vasculitis. Hum Pathol 56:31-39, 2016.

43. Yates $M$, Watts RA, Bajema IM, et al. EULAR/ERA-EDTA recommendations for the management of ANCA-associated vasculitis. Ann Rheum Dis 75:1583-1594, 2016.

44. Ricklin D, Mastellos DC, Reis ES, Lambris JD. The renaissance of complement therapeutics. Nat Rev Nephrol 14:26-47, 2018.

45. Cicardi M, Suffritti C, Perego F, Caccia S. Novelties in the diagnosis and treatment of angioedema. J Investig Allergol Clin Immunol 26:212-221, 2016.

46. Wong EK, Kavanagh D. Anticomplement C5 therapy with eculizumab for the treatment of paroxysmal nocturnal hemoglobinuria and atypical hemolytic uremic syndrome. Transl Res 165:306-320,2015.

47. Legendre CM, Licht C, Loirat C. Eculizumab in atypical hemolytic-uremic syndrome. N Engl J Med 369:1379-1380,2013. 
48. Lewis LA, Ram S. Meningococcal disease and the complement system. Virulence 5:98-126, 2014.

49. Huugen $\mathrm{D}$, van Esch $\mathrm{A}$, Xiao $\mathrm{H}$, et al. Inhibition of complement factor $\mathrm{C} 5$ protects against antimyeloperoxidase antibody-mediated glomerulonephritis in mice. Kidney Int 71:646-654,2007.

50. Schreiber A, Xiao H, Jennette JC, et al. C5a receptor mediates neutrophil activation and ANCA-induced glomerulonephritis. J Am Soc Nephrol 20:289-298,2009.

51. Xiao H, Dairaghi DJ, Powers JP, et al. C5a receptor (CD88) blockade protects against MPOANCA GN. J Am Soc Nephrol 25:225-231,2014.

52. Horiuchi T, Tsukamoto H. Complement-targeted therapy: development of C5- and C5a-targeted inhibition. Inflamm Regen 36:11,2016.

53. Bekker P, Dairaghi D, Seitz L, et al. Characterization of pharmacologic and pharmacokinetic properties of CCX168, a potent and selective orally administered complement 5 a receptor inhibitor, based on preclinical evaluation and randomized phase 1 clinical study. PLoS One 11:e0164646,2016.

54. Jayne D, Bruchfeld AN, Harper L, et al. Randomized trial of C5a receptor inhibitor avacopan in ANCA-associated vasculitis. J Am Soc Nephrol 28:2756-2767,2017.

55. Merkel PA, Niles J, Jimenez R, et al. A randomized clinical trial of CCX168, an orally administered C5AR inhibitor for treatment of patients with ANCA associated vasculitis. Arthritis and Rheumatology 2016; Conference

56. ADVOCATE trial. U.S. National Library of Medicine. ClinicalTrials.gov.at https://clinicaltrials.gov/ ct2/show/NCT02994927. [Accessed 26 March 2019] 